\title{
G0 beam quality and multiple linear regression corrections
}

\author{
Kazutaka Nakahara \\ University of Illinois at Urbana-Champaign 1110 West Green St. Urbana, IL 61801, USA e-mail: nakahara@jlab.org
}

Received: 15 October 2004 / Published Online: 8 February 2005

(C) Società Italiana di Fisica / Springer-Verlag 2005

\begin{abstract}
The G0 experiment measures parity-violating elastic e-p scattering asymmetries to probe the strange quark content of the nucleon. The goal is to measure the asymmetries with an overall uncertainty of $5 \%$ of the measured asymmetries which will be of order $10^{-5}$ to $10^{-6}$. In order to achieve the above precision, systematic effects which can induce false asymmetries must be controlled. One such systematic error is helicity-correlated changes in beam parameters, which, coupled with the sensitivity of the G0 spectrometer to such beam variations, can induce false asymmetries. Beam parameters monitored for helicity-correlation are beam current, beam position, beam angle, and beam energy. A feedback loop was successfully used to reduce the helicity-correlation in beam current and beam position. The sensitivity of the G0 spectrometer to fluctuations in beam parameters has also been measured, and the false asymmetries have been determined to be of order $10^{-8}$. This contribution will address the sensitivity of the G0 spectrometer to changes in beam conditions, the performance of the feedback loops as well as the resulting parity quality of the G0 beam, and the resulting false asymmetry.
\end{abstract}

PACS. 29.27.Hj Polarized beams - 13.60.-r Photon and charged-lepton interactions with hadrons - 25.30.-c Elastic electron scattering

\section{Introduction}

The physics process responsible for the asymmetry measured in the G0 experiment is the weak parity-violating amplitude in elastic scattering [1].

$$
\mathbf{A}=\left(\sigma_{+}-\sigma_{-}\right) /\left(\sigma_{+}+\sigma_{-}\right)
$$

where $\sigma_{+/-}$are the elastic e-p cross sections in the positive/negative helicity states of the electron beam. Thus, the measured asymmetries are susceptible to helicity correlated fluctuations in beam conditions. These fluctuations can alter the measured cross section in a manner unrelated to the physics process we wish to probe, and consequently appear as unwanted false asymmetries. As parity-violation experiments strive to achieve greater precision in the measurements of their asymmetries, the tolerance on helicity-correlated fluctuations in beam conditions have become more stringent. However, recent developments in improving beam quality for such experiments have yielded significant results in reducing false asymmetries coming from helicity-correlated beam fluctuations. These developments, together with the standard multiple linear regression analysis techniques, have rendered such false asymmetries to be a well-controllable systematic effect [2,3]. Although there have been many improvements made both in controlling the helicity-correlation at the source as well as through improved beam transport, this contribution will detail the methods specifically used by the G0 collaboration to reduce these false asymmetries.

\section{Parity quality beam}

Processes which can induce helicity-correlation in the beam may include effects such as an imperfect circular polarization of the laser, helicity-correlated laser motion at the cathode, and beam loading effects in the accelerating cavities 4. In the G0 experiment the cumulative effect from all such processes was measured, and an active feedback device was used to null out the resulting helicitycorrelated differences. An IA (intensity attenuator) cell and a PZT (piezo-electric transducer) mirror were used to modulate the intensity and position of the laser at the cathode in order to null out any charge asymmetry and position differences that exist within the beam. Existing correlations between charge, position, angle and energy made it possible to reduce all 6 helicity-correlated differences simply by feeding back on the above 3 parameters, although it was not guaranteed that the energy difference would be reduced. A halfwave plate was inserted/retracted at the source to reverse the circular polarization of the laser every few days to monitor any further systematics effects coming from polarization reversal. 


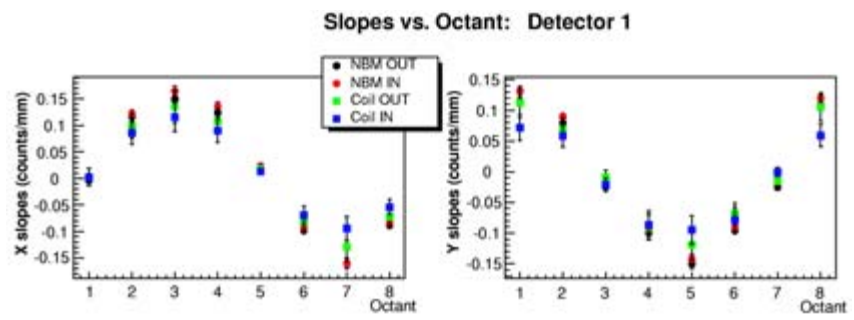

Table 1. The false asymmetries from the helicity-correlated differences as well as the sensitivity of the spectrometer
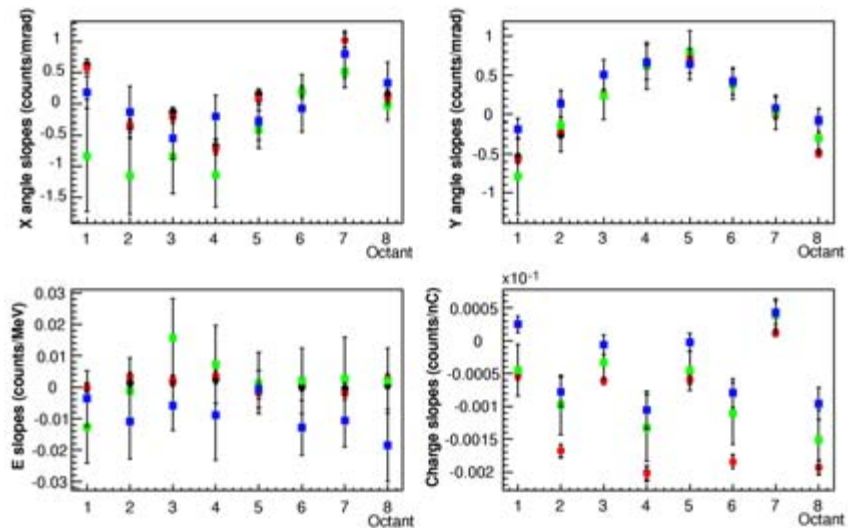

Fig. 1. Linear regression slopes determined from natural beam motion (NBM) and coil modulation show consistent results. The beam current slopes shows a large odd vs even octant dependence due to differing deadtimes from the 2 distinctly separate electronics that were used in those octants

\section{Multiple linear regression}

In order to determine how much false asymmetry results from the helicity-correlation in the beam, the sensitivity of the G0 spectrometer to changes in beam parameters was measured. The false asymmetry resulting from the helicity-correlated parameter differences is thus, 2]

$$
A_{\text {false }}=\Sigma_{i} 1 / Y *\left(d Y / 2 d P_{i}\right) * \delta P_{i}
$$

where $Y$ is the yield seen on the detectors, $\delta P_{i}$ denotes the helicity-correlated differences in the six beam parameters, and $d Y / d P_{i}$ is the slope which characterizes the sensitivity of the spectrometer yield to fluctuations in the beam. The spectrometer is composed of 8 azimuthally symmetric octants, each with an array of 16 detectors which corresponds to different $Q^{2}$, so the false asymmetry contribution due to position and angular motion of the beam tends to cancel out when summed over all octants. In addition to determining the slopes with the natural motion of the beam, a set of steering coils were used upstream of the target to modulate the beam by large amounts in order to gain more dynamic range in determining the sensitiv-

\begin{tabular}{llll}
\hline $\begin{array}{l}\text { Beam } \\
\text { Parameter }\end{array}$ & $\begin{array}{l}\text { Helicity } \\
\text { correlated } \\
\text { difference } \\
\text { (IN-OUT) }\end{array}$ & $\begin{array}{l}\text { Slopes (octant } \\
\text { dependent) }\end{array}$ & $\begin{array}{l}\text { False } \\
\text { asymmetry } \\
\text { (octant-sum) }\end{array}$ \\
\hline $\mathrm{X}$ & $6 \pm 4 \mathrm{~nm}$ & -1 to $1 \% / \mathrm{mm}$ & $10^{-9}$ \\
$\mathrm{Y}$ & $8 \pm 4 \mathrm{~nm}$ & -1 to $0.8 \% / \mathrm{mm}$ & $10^{-9}$ \\
$\theta_{x}$ & $2 \pm 0.3 \mathrm{nrad}$ & -6 to $8 \% / \mathrm{mrad}$ & $10^{-8}$ \\
$\theta_{y}$ & $3 \pm 0.5 \mathrm{nrad}$ & -4 to $5 \% / \mathrm{mrad}$ & $10^{-8}$ \\
$\mathrm{E}$ & $58 \pm 4 \mathrm{eV}$ & -0.01 to $0.02 \% / \mathrm{MeV}$ & $10^{-9}$ \\
$\mathrm{I}$ & $-0.28 \pm 0.28 \mathrm{ppm}$ & $\sim 0^{-3} \% / \mathrm{nC}$ & $10^{-8}$ \\
\hline
\end{tabular}

ity of the spectrometer to beam position and angle. The two methods have shown consistent results throughout the run and the expected octant-dependence is seen. Figure 1 shows the determined sensitivity to changes in the 6 beam parameters for one particular detector element. The resulting false asymmetries coming from the slopes and the helicity-correlation in the 6 beam parameters are summarized on Table1.

\section{Conclusions}

The parity beam feedback system for the G0 forward angle production was successfully implemented, and parity quality beam was achieved to the level where the resulting false asymmetry of order $0.01 \mathrm{ppm}$ is considered a negligible contribution to the $5 \%$ overall uncertainty that the G0 experiment aims for in the determination of its parityviolating asymmetries.

Acknowledgements. Thanks to Doug Beck, Mark Pitt, Matt Poelker, Joe Grames, Yu-Chu Chao, Reza Kazimi, and the Jefferson Lab HallC and Accelerator groups for their tireless effort and support.

\section{References}

1. Douglas H. Beck, Barry R. Holstein: International Journal of Modern Physics E (2001), p. 5-10

2. Damon Spayde: Thesis at the U. of Maryland, Measurement of the Strange Magnetic Form Factor of the Proton using Elastic Electron Scattering, (2001) p. 55 and p. 101-117

3. K.A. Aniol, et al.: Phys. Rev. C 69, 065501 (2004), p. 4-6

4. P.A. Souder et al.: Phys. Rev. Lett. 65, 694 (1990), p. 695697 